\title{
Molecular Characterization of Chikungunya Virus in Serum- Relevance for Disease Management
}

\author{
Komal Gupta ${ }^{1,3}$, Vijay Kumar ${ }^{6}$, Ghanshyam $^{4}$, Narotam Sharma*1, Anita Rawat ${ }^{2}$, Ruchi Agarwal ${ }^{1,5}$, Shiny Saha1 ${ }^{1}$ and \\ Neelam Garg 4 \\ ${ }^{1}$ Central Molecular Research Laboratory, SGRR University, India
}

${ }^{2}$ Uttaranchal PG College of Biomedical Sciences and Hospital, India

${ }^{3}$ Kanya Gurukul Campus, Gurukul Kangri University, India

${ }^{4}$ Kurukshetra University, India

${ }^{5} \mathrm{GEU}$, India

${ }^{6}$ OPJS University, India

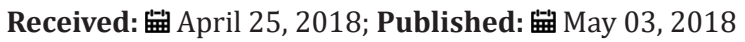

*Corresponding author: Narotam Sharma, Central Molecular Research Laboratory, SGRR University, India,

Email: sharmanarotam5@gmail.com

\begin{abstract}
Background: Chikungunya Virus is a single stranded RNA virus of the genus alphavirus. The transmission of this virus occurs by the bite of infected mosquitoes. It is characterized by an abrupt onset of fever frequently accompanied by joint pain.

Aim: Molecular Characterization of Chikungunya Virus in Serum- Relevance for Disease Management.

Methods and Materials: 30 cases were considered having symptom of fever suspected to have chikungunya. RNA was isolated from magnetic beads methods which were further studied for Real Time-PCR (RT-PCR).

Results: Among the 30 cases suspected for chikungunya fever, only 18 were positive for the same. Hence, the prevalence was calculated to be $60 \%$. About $39 \%$ of the positive cases were in the age range of $21-40$ years, which is the active age group of the total positive cases, $66.66 \%$ were males and $33.33 \%$ were females. The virus can be even transmitted together as a co-infection. Patients infected with chikunguniya virus are more likely to experience symptoms of high fever, severe polyarthralgia and rash with lymphopenia.
\end{abstract}

Keywords: Chikunguniya; Real time polymerase chain reaction; Aedes aegypti; Aedes albopictus

Abbreviations: CHIK V: Chikungunya Virus; CMRL: Central Molecular Research Laboratory; SGRRIM \& HS: Shri Guru Ram Rai Institute of Medical \& Health Science; CDNA: Complementary DNA; MRNA: Messenger RNA

\section{Introduction}

Chikungunya Virus (CHIKV) is an alphavirus with a positive sense single-stranded RNA genome of approximately $11.6 \mathrm{~kb}, 60$ $70 \mathrm{~nm}$ diameter capsid and a phospholipid envelope. It is sensitive to desiccation and to temperatures above $58^{\circ} \mathrm{C}$. It is a member of the Semliki Forest Virus complex. The transmission of this virus occurs with the bite of infected mosquitoes of the genus Aedes. In addition, blood born transmission is possible [1-3]. The incubation

period ranges from 3 to 12 days. The onset is usually abrupt and the acute stage is characterized by sudden high fever, incapacitating arthralgia, myalgias and skin rash. Chronic arthritis may develop in the patients and is associated with fever, asthenia and exacerbation of arthralgia, inflammatory polyarthritis, and stiffness $[4,5]$. The Alphavirus group comprises of 28 viruses, six of which can cause human joint disorders-namely chikungunya virus, o'nyong-nyong 
virus (central Africa), Ross River and Barmah Forest viruses (Australia and the Pacific), Sindbis virus (cosmopolitan), and Mayaro virus (South America, French Guyana). These alphaviruses share certain antigenic determinants [6-9].

\section{Materials and Methods}

30 clinical samples were considered in this study. Blood samples were taken from the suspected cases of Chikungunya from the different departments of Shri mahant Indiresh Hospital, dehradun (Uttarakhand) and further processed at Central Molecular Research Laboratory (CMRL), Shri Guru Ram Rai Institute of Medical \& Health science (SGRRIM \& HS), Patel nagar, dehradun (U.K.) for the molecular characterization of Chikungunya Virus.

\section{Collection of EDTA Blood \& Separation of Serum/Plasma}

Whole blood samples were centrifuged at 5,000rpm for 10 15 minutes at $4^{\circ} \mathrm{C}$. The serum was separated from blood \& then subjected for nucleic acid extraction. Then Isolation of RNA for the detection of chikungunya virus was done by magnetic beads method where Magnetic carriers bearing an immobilized affinity or hydrophobic ligand or ion exchange groups or magnetic biopolymer particles having affinity to the isolated structure are mixed with the sample containing target compounds. The specimens with target nucleic acid are processed by lysis buffer and then the nucleic acids are efficiently bound to the specifically modified magnetic beads. The beads were removed from the solution and attached to the tube wall manually using a magnetic separator stand. After washing, purification and elution, ultra-purified RNA can be obtained. After RNA was obtained master mix was prepared for chikungunya RNA for qualitative detection by Real Time PCR. Reverse transcription was done (RT-PCR) for the extracted RNA specimens. In this method, RNA was first transcribed into complementary DNA (cDNA) by reverse transcriptase from total RNA or messenger RNA (mRNA). The cDNA is then used as the template for the qPCR reaction.

\section{Results}

Among the 30 cases suspected for chikungunya fever, only 18 were positive for the same. Hence, the prevalence was calculated to be $60 \%$. About $39 \%$ of the positive cases were in the age group ranging in between 21-40 years, which is the active age group of the total positive cases, $66.66 \%$ were males and $33.33 \%$ were females. Ct value in molecular profiling indicates that the sample is positive for chikungunya viral genome whereas no $\mathrm{Ct}$ value is indication of negative results for chikungunya. Ct value for internal control was in the range of 14-17 shows that the result is valid (Figure 1).
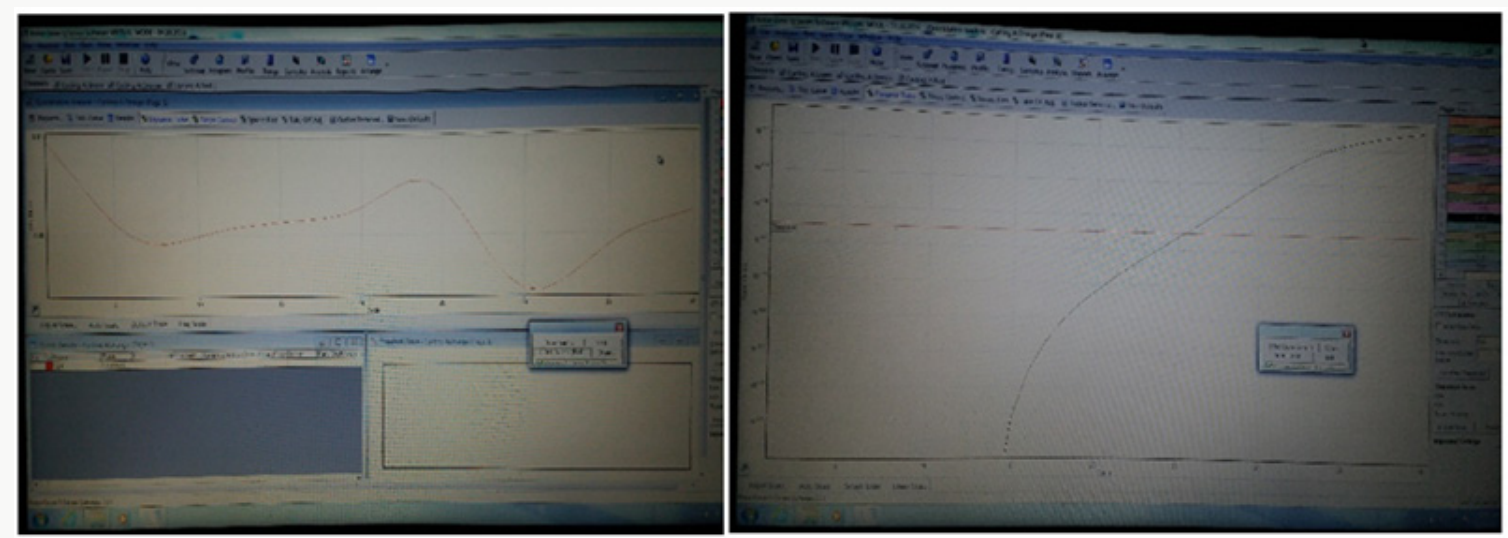

Figure 1: Amplification curves in Real time PCR for Chikungunya virus.

\section{Discussion and Conclusion}

Chikungunya (CHIKV) is a mosquito-borne disease caused by an RNA alphavirus of the Togaviridae family. The main mosquito vectors are the aggressive Aedesaegypti and Aedesalbopictus. The most common presentation of CHIKV is acute onset of fever and polyarthralgia, sometimes followed by a macupopular rash. Other associated symptoms can include headache, myalgia, nausea and vomiting. These symptoms typically occur 3 to 7 days after the mosquito bite and generally last 7 to 10 days. Rare complication of CHIKV can include uveitis, retinitis, myocarditis, hepatitis, nephritis, bullos skin lesions, hemorrhage, meningoencephalitis. The clinical manifestations of CHIKV can be very similar to dengue fever, which is transmitted by the same species of the mosquito. The 2 viruses can be even transmitted together as a co-infection. Patients infected with CHIK are more likely to experience symptoms of high fever, severe polyarthralgia and rash with lymphopenia, whereas patients with dengue fever are more likely to have symptoms of hemorrhage, shock and death with associated laboratory derangements of neutropenia and thrombocytopenia. However, because of the close similarities, dengue fever should be strongly considered in the differential diagnosis of patients suspected with CHIK. Conformational testing for CHIK can be performed in one of the three ways. These methods include viral culture if tested within first three days of illness, PCR in the first eight days, or antibody serology after the first three days of illness. Other laboratory abnormalities may include lymphopenia, thrombocytopenia, elevated creatinine and elevated hepatic transaminases. Treatment is primarily supportive. The preventive measure among the individual level included the 
use of mosquito repellents like coils, body creams, mosquito nets, etc. At the community level, it is important to ensure that there are no collections of water in household vessels or around dwelling places. The usual recommendation is that on every fifth day, all the vessels, which contain water, should be emptied; this observance of a dry day breaks the life cycle of the mosquito [10].

From above discussion, we conclude that, Chikungunya fever is self-limiting, the morbidity can be very high in major outbreaks, resulting in heavy social and economic tolls. The prevention of the disease requires a planned approach, besides knowledge and awareness on the early warning signs. An integrated vector management through the elimination of the breeding sites, the use of anti-adult and anti-larval measures and personal protection will contribute to the prevention of outbreaks. A community empowerment and mobilization is crucial for the prevention and control of Chikungunya.

\section{Acknowledgement}

The authors are grateful to Honorable Chairman, Shri Guru Ram Rai Education Mission for his kind support, guidance and favor.

\section{References}

1. Khan AH, Morita K, Parquet MdMdel C, Hasebe F, Mathenge EG, et al. (2002) Complete nucleotide sequence of chikungunya virus and evidence for an internal polyadenylation site. J Gen Virol 83(12): 30753084 .
2. Strauss JH, Strauss EG (1994) The alphaviruses: Gene expression, replication, and evolution. Microbiol Rev 58(3): 491-562.

3. Yadav P, Shouche YS, Munot HP, Mishra AC, Mourya DT (2003) Genotyping of chikungunya virus isolates from India during 1996-2000 by reverse transcription-polymerase chain reaction. Acta Virol 47(2): 125-157.

4. Weaver Scott C, Marc Lecuit (2015) Chikungunya virus and the global spread of a mosquito-borne disease. New England Journal of Medicine 372(13): 1231-1239.

5. Cavrini Francesca, Paolo Gaibani, Anna Maria Pierro, Giada Rossini, Maria Paola Landini, et al. (2009) Chikungunya: An emerging and spreading arthropod-borne viral disease. The Journal of Infection in Developing Countries 3(10): 744-752.

6. Rigau Pérez José G, Gary G Clark, Duane J Gubler, Paul Reiter, Eduard J Sanders, et al. (1998) Dengue and dengue haemorrhagic fever. The Lancet 352(9132): 971-977.

7. Pacheco Oscar, Mauricio Beltrán, Christina A Nelson, Diana Valencia, Natalia Tolosa, et al. (2016) Zika virus disease in Colombia-preliminary report. New England Journal of Medicine.

8. Prat Christine M, Olivier Flusin, Amanda Panella, Bernard Tenebray, Robert Lanciotti, et al. (2014) Evaluation of commercially available serologic diagnostic tests for chikungunya virus. Emerging infectious diseases 20(12): 2129-2132.

9. Grandadam Marc, Valérie Caro, Sébastien Plumet, Jean Michel Thiberge, Yvan Souares, et al. (2011) Chikungunya virus, southeastern France. Emerging infectious diseases 17(5): 910-913.

10. Simon Fabrice, Emilie Javelle, Manuela Oliver, Isabelle Leparc Goffart, Catherine Marimoutou (2011) Chikungunya virus infection. Current infectious disease reports 13(3): 218.

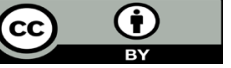

This work is licensed under Creative Commons Attribution 4.0 License

To Submit Your Article Click Here: Submit Article

DOI: 10.32474/AOICS.2018.02.000148

\section{AOICS}

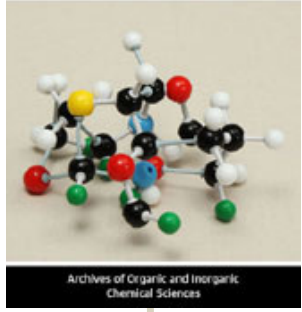

Archives of Organic and Inorganic Chemical Sciences

\section{Assets of Publishing with us}

- Global archiving of articles

- Immediate, unrestricted online access

- Rigorous Peer Review Process

- Authors Retain Copyrights

- Unique DOI for all articles 